\title{
Automatic recognition of context and stress to support knowledge workers
}

\author{
Saskia Koldijk \\ TNO \& Radboud University Nijmegen \\ The Netherlands \\ s.koldijk@cs.ru.nl
}

Supervisors: Wessel Kraaij and Mark Neerincx

\begin{abstract}
Motivation - Developing a computer tool that improves well-being at work.

Research approach - We collect unobtrusive sensor data and apply pattern recognition approaches to infer the context and stress level of the user. We will develop a coaching tool based upon this information and evaluate its effectiveness in user studies.
\end{abstract}

Findings/Design - The resulting system will be able to create more awareness on stress for knowledge workers, support them to improve their working pattern, resulting in an increase of well-being at work.

Take away message - Unobtrusive sensing and smart reasoning can be used to create a user aware system that improves well-being at work by providing feedback and support.

\section{Keywords}

Knowledge worker, stress, workload, unobtrusive sensing, pattern recognition, feedback, personalization.

\section{INTRODUCTION}

Stress is a common experience nowadays. For knowledge workers, who are predominantly concerned with interpreting and generating information, stress is easily caused by their typical working conditions (Michie, 2002). They get overwhelmed by information, work under high demands and often have a fragmented way of working due to interruptions. As a consequence, well-being at work can be declined, which may finally result in burn-out.

We see the computer, which is often used at work, as important means to address well-being at work. Much information about the user's current context and state can be captured. Based on this information, the computer can help the user by providing feedback and support that is optimally adapted to the situation and state of the user. Feedback can create more awareness of the mental state and possible underlying causes. By providing support, for example with the planning of activities or providing useful information just in time, mental workload and stress can be kept in optimal ranges.
In general, well-being at work is often approached from an organizational point of view. With questionnaires like the NOVA-WEBA (Kraan, Dhondt, Houtman, Nelemans \& Vroome, 2000), employees are asked to rate various aspects of their work. The results of these questionnaires are then used to re-organize the work. In our research, we want to enable real-time measuring of relevant aspects of well-being at work. This information can then be directly acted upon. Knowledge workers work relatively autonomously, so we see much potential for them to contribute to the improvement of their own well-being.

Using technology for improving well-being has many advantages (Ijsselsteijn, de Kort, Midden, Eggen \& van den Hoven, 2006), e.g. its persistence or objectiveness, the possibility to provide just-in-time notifications with relevant, actionable information or their supportive and motivating role. Advancements in sensing and interpretation are described as promising and it is noted that context sensing and appropriate feedback are important areas for research. In our work we will address these aspects.

Figure 1 depicts our basic underlying framework. The knowledge worker behaves in a certain way, which can be captured by various sensors. This sensor data will then be interpreted. Pattern recognition approaches will be applied to gain insightful information from the low level sensor data. This information will then be used to give the user feedback about his or her behaviour and provide support. Tuning the interpretation module to the specific user and adapting feedback and support to the users context and mental state are typical aspects for cognitive ergonomics. Advancements in the state of the art will be achieved in the areas of multimodal sensor integration, contextual reasoning, activity and task recognition, mental and physical state estimation and user adaptation through learning.

In the remainder of this paper, we will outline our work regarding the following 3 aspects:

- Requirements for the tool

- Automatic recognition of context and user state

- Developing feedback and support 
We describe these aspects in more detail, specify the research methodologies we use, present some initial results, as well as planned work.

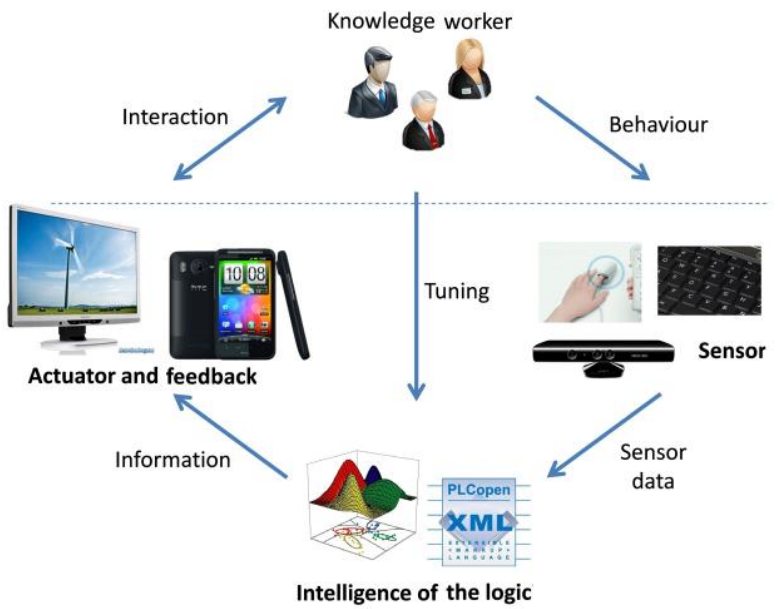

Figure 1. General framework for providing feedback and support.

\section{REQUIREMENTS}

The first question to answer is: which aspects need to be considered for making an effective coaching tool? Literature and user input are used to formulate a set of requirements.

\section{Determinants for well-being at work}

We want to develop a computer tool that can help people to better cope with the negative determinants of well-being. Preferably it should be possible to capture relevant factors by sensing interactions with a computer. In a literature study we investigated the determinants for well-being at work and decided to focus on the factors of the work itself and the working conditions.

\section{Questionnaire}

In order to get insights in the working style and needs of knowledge workers, we developed a questionnaire. In total 47 employees from TNO (Netherlands Organization for Applied Scientific Research) with various backgrounds and different functions responded.

The answers reveal that the knowledge workers spend a great amount of their time $(66.7 \%)$ at the pc and are very autonomous in managing their working time, which makes our approach suitable. They are typically involved in several different projects at a time (avg: 5.3) and have to manage various deadlines, which indicates that good self-management plays an important role. An essential part of the work performed is dependent on other people (mailing, meeting, calling, together $41 \%$ of their time). This requires that the work is well planned. Regarding the own work planning, 50\% of the knowledge workers indicate to work reactively. This means for many workers the course of actions over a day is not self-determined which might cause a lack of overview or a feeling of stress.
There seems to be no clear preference for working style among knowledge workers. Some prefer to focus on one task whereas others like to switch tasks. Moreover, there are individual differences whether interruptions are perceived as annoying. This is important to know for individualizing the software toward the specific user.

Furthermore, the responses show that recognition on the level of tasks is perceived as more useful than simple application logs. It is important that the system aggregates and interprets low level data and links actions to projects to yield the user useful information. Typical functionality suggested is enabling a comparison of ones activity with the planned activity or the personal average. General tips concern the reliability and flexibility of the system. Most concerns respondents have are about privacy and losing control.

In general the results confirm our view on knowledge workers. The given answers help us to focus our research towards important aspects, like high level interpretation of data and personalization of the tool.

\section{Persona and use cases}

A workshop with several knowledge workers and domain experts was organized to formulate personas and use cases to give the problem context more detail and develop possible solutions.

A result of this workshop is a persona at risk of burnout, for which we worked out personal characteristics, problems experienced and why she is unable to solve these problems herself. Based upon this description we identified underlying causes of the problems experienced, how the person can be helped to solve the problems and how a computer tool can be used for this.

Based on our gained information, the next step is to formulate a set of requirements for the tool.

\section{RECOGNITION OF CONTEXT AND USER STATE}

The next question to answer is: is it possible to infer relevant aspects of context and user state, based on unobtrusive sensing? We now describe the underlying models, sensors to be used and the recognized contexts and user states in more detail.

\section{Model of sensors to concepts}

In order to automatically recognize the relevant aspects of the user context and the mental state of the user, we take a pattern recognition approach. From sensor data, specific features will be extracted which are provided to a classifier to assign an interpretation label. A first setup for linking different sensors to contexts and user states was made based on a literature study.

In Figure 2 you can see that various sensors on the computer can be used for recognizing different aspects of context. The task the user is performing (e.g. writing a report or making a presentation) could be recognized on basis of keyboard, mouse and application information. The content context (e.g. the project worked on) could be determined based upon the accessed content. The social context of the employee could be given by mail and phone conversations. 
Finally, aspects of the physical context, like location, level of activity, posture or noise level, could be inferred from GPS, accelerometers, visual and audio information.

The combination of tasks and contents worked on can give insights in the experienced workload. In combination with the social and physical context this can be used for an estimate of stress.

The emotional state, in terms of valence and arousal, could be estimated based on video or audio, or using additional body sensors measuring respiration, heart signal (ECG), skin response (SCR) or blood volume pulse (BVP). These valence and arousal estimates can give additional information on the experienced stress level. Furthermore, useful information for the model could be gained by asking the user for input, for example asking whether he is in a good or bad mood.

As mentioned, this is a first setup which we will need to adapt and extend, based on new insights gained.

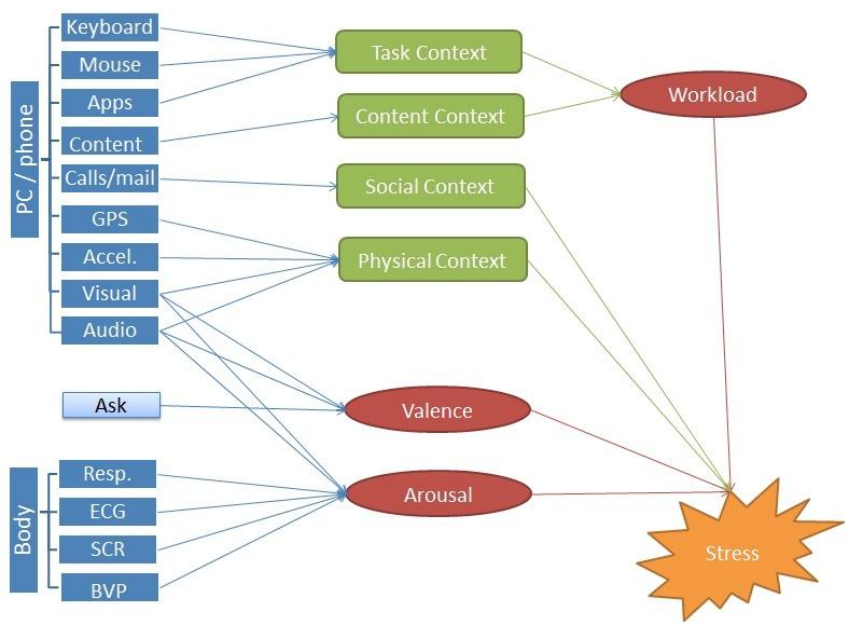

Figure 2. First setup for linking different sensors to contexts and user states.

\begin{abstract}
User model
The model described in the previous section will be used as basis for every user. Nevertheless it is important to note that each person is different and thus the relations between the different aspects may vary per user. The same task can for example have a lower or higher workload for a knowledge worker depending on his level of experience and expertise. Also well-being is subjective, and so personal characteristics are important to consider.
\end{abstract}

Therefore a user model will be learned for each user. Steps to take are selecting relevant characteristics to store and how to acquire them.

\section{Data collection}

In order to train and test our recognition models, data from several knowledge workers needs to be collected during their working day and annotated with context labels.
First experiences with collecting data in real-world office environments shows that colleagues are willing to participate, but the annotations require much effort and motivation. Giving participants something in return seemed to be a good approach, for example providing overviews of recognized activities or embedding data collection in a game.

Currently a database to collect large amounts of sensor data is set up. The next step is preparing a good set of annotation labels, implementing a user friendly data collection tool and collecting data from knowledge workers.

\section{Context recognition}

Regarding the recognition of the different context aspects, first results for the recognition of tasks were already obtained (Koldijk, van Staalduinen, Neerincx \& Kraaij, 2012).

Our research has shown that task recognition on the basis of PC activity is challenging but feasible. Unlike other research, in which clearly structured tasks were modelled (e.g. Natarajan, Bui, Tadepalli, Kersting \& Wong, 2008), our research has shown that task recognition also works for less structured tasks and more spontaneous activity, since our results were obtained using realistic data.

Task recognition is very personal, as different users have different work styles and task mixes. Nevertheless, we saw that on an individual basis, the simple classifiers we used learn to recognize tasks quite fast, yielding a performance up to $80 \%$ which is reasonable high, considering 12 possible task labels that were used.

Finally, since different users show different patterns of behaviour when performing a task, the classification model should be trained for each specific user to yield optimal task recognition. We concluded that no more than 2.5 hours (30 instances) of representative training examples is required to train a good model.

These results are very promising. Next, we will address the recognition of other aspects of context. We will define these contexts in greater detail and select the most useful sensors and features. Again, the recognition performance and learning speed of several recognition approaches will be tested on real-world data.

\section{User state recognition}

Besides recognizing the context, we want to estimate the user state in terms of workload, valence, arousal and finally stress. Therefore, we will have to reason about contexts and user states on a higher level and validate our estimates. Preferably the rather obtrusive body sensors will only be used in our experiments. We hope to find unobtrusive measurements that are correlated with these measures of stress to be used in the final application.

\section{FEEDBACK AND SUPPORT}

The final question to be answered is: which methods of feedback and support are most effective for the user? 
We organized a workshop with several knowledge workers to collect some ideas.

\section{Possibilities for feedback}

To investigate ways for providing feedback, we asked the participants to draw their visualization ideas and afterwards we discussed these sketches.

A participant noted that in the first place simple visualizations should be presented that could be quickly grasped, and that a more detailed overview would be useful later. More metaphorical visualizations included using a traffic light, smiley's or flowers to depict how well the state of working is. More technical visualizations with facts in detail include bar charts and graphics depicting trends over time. The specific visualization preferences turned out to be very different among people, thus personalization is a very important issue.

As next step, various forms of visualizations will be created based upon this input, and tested in user experiments for preference and usefulness.

\section{Possibilities for support}

To investigate ways for providing support, we asked the knowledge workers to think about how to optimally assist them in their self-management.

Answers show that the tool should help them gain insights and discover aspects they were unaware of before. It could help to judge in how far the current situation deviates from the desired, for example warning when the stress level rises or when the working pattern deviates and might indicate a bad way of working. The tool could contrast the planned time with the actual time of completion, which can help to make better time estimates and a more realistic planning. Moreover, the tool could give insights in what kind of days or working styles typically lead to a state of high satisfaction and which do not. It was noted that also positive feedback or compliments are important. Professional tips or best practices of others, e.g. on how to diminish stressors, were appreciated.

Mentioned possibilities to intervene were blocking the calendar when the workload becomes too heavy or filtering incoming emails according to their importance. The tool could also protect the user's flow, by blocking disturbing factors. Regarding the change of behaviour, users could formulate goals to reach, for example to work less or empty the inbox regularly, or indicate which activities have priority for them. Users would like to know whether one's own norms are realistic, which could be done by comparing oneself with a benchmark based on other user's data. Also some games to help improving behaviour were suggested, for example a flow meter.

The next steps we will take are developing suitable ways for support based upon these initial ideas and literature on behaviour change and stress. These will then be tested in user experiments for their appropriateness.

\section{Learning of suitable methods}

Every user is different, so the tool should learn from the behaviour of the user whether the applied strategy works, in order to guarantee long lasting success, without irritating the user. Steps to take are generating a system that applies various coaching strategies, and learns from explicit and implicit feedback from the user.

\section{Human computer interaction}

For long lasting success of the tool also the interaction of the system is of importance. Steps to take are developing one or more user interfaces and test them for their user friendliness.

\section{CONCLUSION}

We presented our research plans and first results for a tool to improve well-being at work. Main aspects are recognizing context and user state and providing feedback and support based upon this information. Personalization of the tool and evaluation in real-world settings is seen as very important.

Our initial result demonstrate the feasibility of our approach of collecting and interpreting data of realworld office settings. It is important to note that the correct and robust interpretation of this data is essential for the coaching tool. In case no satisfying results regarding automatic recognition can be obtained, smart questions to the user will be used to enrich the information the computer has.

To conclude, this research will result in insights regarding the possibilities to improve well-being at work by means of unobtrusive sensing and automatic interpretation of data.

\section{ACKNOWLEDGMENTS}

This publication was supported by the Dutch national program COMMIT (project P7 SWELL).

\section{REFERENCES}

IJsselsteijn, W., de Kort, Y., Midden, C., Eggen, B., van den Hoven, E. (2006). Persuasive technology for human well-being: setting the scene. In: Proceedings of PERSUASIVE 2006, pp 1-5.

Koldijk, S., van Staalduinen, M., Neerincx, M., Kraaij, W. (2012). Real-time task recognition based on knowledge workers' computer activities. Proceedings of ECCE 2012 (Edinburgh, Scotland, 28-31 August 2012).

Kraan, K.O., Dhondt, S., Houtman, I.L.D., Nelemans, R. \& Vroome, E.M.M. de (2000). Handleiding NOVA-WEBA: Hernieuwde versie. Hoofddorp: TNO Arbeid.

Michie, S. (2002). Causes and management of stress at work. Occup. Environ. Med., 59, 67-72.

Natarajan, S., Bui, H., Tadepalli, P., Kersting, K., \& Wong, W. (2008). Logical hierarchical hidden markov models for modeling user activities. In Proc. of ILP-08, pp 192-209. 